\title{
Investigating the Impact of Feature Reduction Through Information Gain and Correlation on the Performance of Error Back Propagation Based IDS
}

\author{
Ghanshyam Prasad Dubey ${ }^{1}$ and Dr. Rakesh Kumar Bhujade ${ }^{2}$ \\ ${ }^{1} R S$, DoCSE, Mandsaur University, India \\ ${ }^{2}$ Head, DoIT, Govt. Polytechnic, Daman, India \\ *Correspondence: Dr. Rakesh Kumar Bhujade; Email: rakesh.bhujade@ gmail.com
}

\begin{abstract}
Research in the field of IDS has been going on since long time; however, there exists a number of ways to further improve the efficiency of IDS. This paper investigates the performance of Intrusion detection system using feature reduction and EBPA. The first step involves the reduction in number of features, based on the combination of information gain and correlation. In the next step, error back propagation algorithm (EBPA) is used to train the network and then analyze the performance. EBPA is commonly used due to its ease of use, high accuracy and efficiency. The proposed model is tested over the KDD Cup 99 and NSL-KDD datasets. Results show that the proposed IDS model with reduced feature set outperforms the other models, both in terms of performance metrics and processing time.
\end{abstract}

Keywords: Intrusion Detection, Back Propagation, Feature Selection, Machine Learning, KDD-99.

\begin{tabular}{|c|c|}
\hline \multicolumn{2}{|l|}{ ARTICLE INFORMATION } \\
\hline \multicolumn{2}{|c|}{$\begin{array}{l}\text { Author(s): Prof. Ghanshyam Prasad Dubey, Dr. Rakesh Kumar Bhujade } \\
\text { Received: } 27 \text { Sep, 2021; Accepted: } 10 \text { Dec, 2021; Publis hed: } 17 \text { Dec, 2021; }\end{array}$} \\
\hline $\begin{array}{l}\text { e-ISSN: } 2347-470 X \\
\text { Paper Id: IJEER-2021-07; }\end{array}$ & crossiref member \\
\hline $\begin{array}{l}\text { Citation: https://doi.org/10.37391/IJEER.090302 } \\
\text { Webpage-link: }\end{array}$ & $\begin{array}{l}\text { CROSSREF.ORG } \\
\text { THECTATIONLINKING BACKBONE } \\
\end{array}$ \\
\hline
\end{tabular}

\section{INTRODUCTION}

Recent technological developments led to a significant rise in the number of anomalies, threats, misuses, and hacks to Resources, Services, and Data by unfair means or unauthorized access has increased significantly. This has necessitated the creation of a solution for preventing, managing, and resolving these misuses in order to ensure that only approved users have access to Resources [1]. Firewalls, Intrusion Prevention Systems abbreviated as IPS, and Intrusion Detection Systems known as IDS are some of the most popular solutions for dealing with the above-mentioned issues [2]. The Intrusion Detection System shall take appropriate action, such as alarm or alert generation, and shall prevent such action. The varieties of the IDS systems are not capable to diagnose the new patterns of attack; some are generating fake alarms, some of them are not able to detect malicious activity [3]. Denning first coined the term "IDS" in 1987[4]. Intrusion Detection Systems are classified based on detection approaches, intrusion response, target systems (where they are located), timing analysis, and architecture (fig. 1).

IDS can be classified in two groups based on their location of installation: first one is host-based intrusion detection system (abbreviated as HIDS) placed on each host or terminal and second one is network-based intrusion detection system (NIDS) [5] [6] applied on servers or gateways.

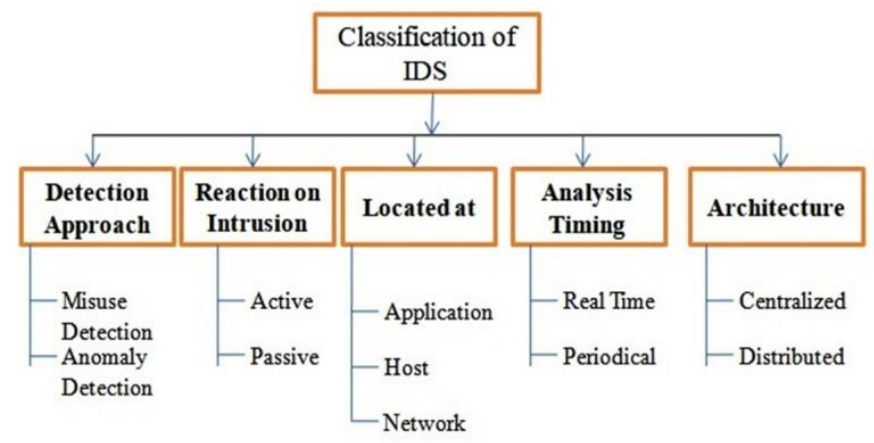

Figure 1: Types of IDS.

An HIDS is a program that is mounted on a host and monitors the activity of that node. A NIDS is a system applied on server or gateway that monitors network traffic and activity for signs of attacks. In terms of current trends, intrusion detection incorporates host-based and network-based knowledge to create hybrid systems that have better performance. These hybrid systems are designed to take advantage of the best of both worlds. Their client versions are used to track host actions and submit a report to the network manager and manager so that they can compare whether or not the behavior is malicious. After diagnosing an attack or a behavioral deviation, it takes action.

A great deal of research has already has been done; even further research is warranted in the field of IDS. In order to develop the Optimized IDS, it is necessary to consider certain issues, including low false alarm rate, high detection rate, effective processing of unbalanced datasets and low response times. Machine Learning techniques resolve most of these issues, as they are highly accurate and have a low False Alarm Rate; at the same time, they can address the problem of 
imbalanced datasets [7]. Machine Learning techniques aid in the improvement of model output while also imparting learning abilities to models, allowing them to learn while in service. Although anomaly detection is important for discovering unknown or new attack patterns, it also presents the issue of False Alarms. Machine learning is frequently used in conjunction with Anomaly-based detection to create an IDS model with high accuracy at the same time, with the resulting danger of a significant False Alarm Rate [8].

\section{FEATURE REDUCTION}

Intrusion Detection Datasets, such as KDD CUP 99 [9], NSL KDD [10], and others, are very large. It will take a long time for a Model to learn if it is trained on this massive dataset. The Model's learning or training time will increase as the size of the Dataset increases. As a result, the issue of reducing the size of the available Dataset in such a way that the resulting reduced Dataset is identical to the original Dataset and all of the insights in the original Dataset are guaranteed for the resultant Dataset arises. Feature Engineering or Dimension Reduction is common terms for this mission. It is very important in the development of a Machine Learning Model [11] [12] (fig.2).

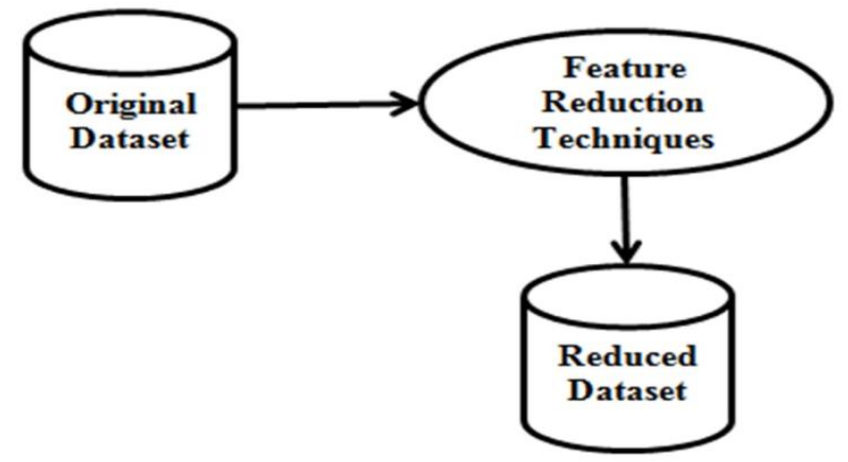

Figure 2: Feature Reduction Concept.

The aim of feature selection is to eliminate redundancy. Relevant Features are included in the resulting Dataset, while redundant features are removed, resulting in a smaller Dataset that can be used to train the model. Metrics like Information Gain, Correlation, Mutual Information, and others among features can be used to identify the optimized feature subset [13]. The resulting Feature subset would include all of the Features from the original Feature Set that have high Relevancy and no or low Redundancy, resulting in effective and efficient model training [14],[52],[53]. H. Lim and D. Kim proposed a method called "Pair-wise Dependence-based Unsupervised Feature Selection." Their method is based on the Information Gain theory [15],[54],[61]. The "Feature Selection for Multi-Label Classification based approach used Clustering in Feature Space," proposed N. Mishra and P. Singh. It is a Wrapper approach that does not has need of parameter tuning and therefore saves time. Sample-based Precision and Recall estimates are used to enforce Feature Ranking [16],[50],[51].

Feature Reduction is an important step in the Data Preprocessing phase, while developing a Classifier. It helps in reducing the overheads associated with computation time and storage requirements, thereby improving the overall performance of the classifier model. In Intrusion Detection Systems (IDS), Feature Reduction techniques improve the accuracy of the Classifier [17]. Principal Component Analysis (PCA), Genetic Algorithms (GA), Information Gain (IG), etc are some common approaches, used for Feature Selection [18].

\section{ERROR BACK PROPAGATION}

The most popular and commonly used Neural Network approach is back propagation [19]. Rumelhart first suggested it in 1986. It's a multi-layer feed forward Neural Network that's been equipped with the Error Back Propagation algorithm [20]. It is a Supervised Learning method in which the Model is trained using Labeled Data [21]. The BPNN is split into two phases: forward and backward. In the forward step, the model receives input and generates an output; the output is then compared to the desired output, and the error is calculated. The Error is propagated in the Network during the Backward step, and the weights of the links connecting nodes are changed, and the input is given to the model again, and output is generated. This process is repeated until the Error reaches 0 or the desired minimum. To increase the approach's efficiency and minimize Error as soon as possible, the Gradient Descent technique is used to compute the Error, resulting in a significant reduction in training time [20].

Scaled Conjugate Gradient, Gradient Descent with Momentum, Adaptive Learning, BFGS, Levenberg Marquardt, One Phase Secant, Bayesian Regularization, and other algorithms can be used to train a Back Propagation Neural Network [22]. The results of various BPNN training functions show that Levenberg Marquardt (LM) is the best training method for Back Propagation Neural Networks [23],[62],[63]. Back Propagation is commonly used due to its ease of use and high accuracy and efficiency. It is commonly used in the development of IDS models.

\section{LITERATURE SURVEY}

Alhaj, Taqwa Ahmed, et al proposes a two tier Feature Selection approach; the first tier is Feature Ranking and the second tier is Additional Feature. Information Gain measure is used to rank the features, according to relevancy and minimal redundancy. Four best features, based on ranking, are selected in the reduced subset of features. Three more features are added in the second tier in this feature subset. Correlation between features is used as measure for identification of additional features in the second tier [24],[55],[56]. Shahbaz, Mahsa Bataghva, et al proposes a Feature Selection technique based on correlation and symmetric uncertainty. The proposed technique has two main steps of operation. In the first step, correlation estimate is used for identifying the suitable subset of features, which are relevant. In the second step, symmetric uncertainty is used to remove the redundant features from the newly formed feature subset, so as to get the optimal set of reduced features [25],[66],[59].

Salih, Azar Abid, and Maiwan Bahjat Abdulrazaq propose a 
Feature Selection technique based on the principle of voting. Three estimates, namely Information Gain, Gain Ratio and Correlation are utilized to identify the most relevant features. Each estimate will compute the set of most relevant features and then two top ranked features from each of these estimates are collected together to generate the feature subset, representing the original dataset [26].

Zhang, Yong, Xuezhen Ren, and Jie Zhang proposed a Feature Selection technique for Intrusion Detection Datasets, based on Information Gain measure and Relief F Algorithm. Relief F is a weighted Filter based Feature Selection approach that works primarily by computing the correlation estimates. It is a threshold based approach, where threshold is applied at Information Gain estimate, as well as at weighted correlation estimate in case of Relief F Algorithm. The approach first computes the information gain estimate for all features. All features where estimate satisfies the threshold are selected as elements of optimal feature subset 1, termed as M1. Now, Relief F Algorithm will compute the weight of each feature using correlation estimate. All features which satisfy the weight threshold of Relief $\mathrm{F}$ are selected as elements of optimal feature subset 2, termed as M2. Lastly, features of M1 and $\mathrm{M} 2$ are combined together as M1 U M2, which reflects the final optimal feature subset [27],[45],[46],[47],[49].

This paper [28] proposes IFSA, a Feature Selection approach for computing an optimal subset of features using Filters and Information Gain measure. IFSA is an Unsupervised Discretization approach that uses information gain as estimate for identifying the relevant and non - redundant features that forms the optimal feature subset, representing the original dataset in an effective manner [28]. Correlation among Features is computed using three types of measures, namely Entropy based Measures (Information Gain, Gain Ratio and Symmetric Uncertainty), Statistical based Measures (Chi Squared) and Instance based Measures (Relief F). This paper proposes a Data Dimensionality Reduction Scheme for Intrusion Detection Systems using Ensemble and Standalone Classifiers. The proposed Dimensionality Reduction scheme incorporates one technique from each type of correlation measure, as Symmetric Uncertainty as Entropy based Filter, Chi Squared as Statistical Measure and Relief F as Instance based Measure [29]. Artificial Neural Network based systems are adopted by numerous researchers for training and testing their models [30], [31], [32].

\section{PROPOSED ALGORITHM}

In this section proposed algorithm (Feature Reduced Back Propagation) is presented. FRBP (Feature Reduced Back Propagation) method divided in to three modules based on their working like data pre-processing, information gain calculation, correlation findings, making of compatible sets to reduced feature, back propagation neural network training and prediction verification etc.

\subsection{Computing Information Gain}

Knowledge Gain is a metric that determines how much information a function provides about a class. Perfectly partitioned features can have the most detail. A function that is unrelated to the main feature should have no detail. For the purpose of calculating knowledge gain, the entropy of each set of classes is measured as follows:

$$
E_{\text {entropy }}=-\sum_{\mathrm{i}=1}^{\mathrm{K}} \mathrm{p}_{\mathrm{k}} \log _{2} \mathrm{p}_{\mathrm{k}}
$$

Where, $\mathrm{p}_{\mathrm{k}}$ denotes the proportion of instances belonging to class $\mathrm{k}(\mathrm{K}=1,2 \ldots . \mathrm{k})$

After calculating entropy of the dataset, change in gain or Information gain of each feature in the dataset can be calculated as

$$
\Delta E=E-\frac{m_{L}}{m} E_{L}-\frac{m_{R}}{m} E_{R}
$$

Where, $\mathrm{m}$ is the total number of instances, with $\mathrm{m}_{\mathrm{k}}$ instances belonging to class $\mathrm{k}$, where $\mathrm{K}=1,2 \ldots \mathrm{k}$

\subsection{Computing Correlation among Features}

A Correlation is a coefficient which is used for some statistical relationship between variables in dataset. The Correlation can be computed as:

$$
M_{s}=\frac{k_{r_{c f}}}{\sqrt{k+k(k-1) r_{f f}}}
$$

Where, Ms is the heuristic merit of a feature subset $\mathrm{S}$ Containing $\mathrm{k}$ features and $\mathrm{rcf}$ is the mean of the feature-class correlation ( $f \in S$ ), and rff means the average feature-feature inter-correlation.

Step 1: Initialize the input training KDD-99 Network Dataset. Step 2: Reduce the Dataset Feature by performing ranking of dataset features by applying Information gain concept along with Correlation.

i. From Dataset ' $D$ ' compute Information gain 'I' of each feature set ' $F$ ' and rank each of the feature from high information gain to low gain as I1, I2, I3, I4....In.

ii. From Dataset ' $\mathrm{D}$ ' compute Correlation ' $\mathrm{C}$ ' of each feature set ' $\mathrm{F}$ ' and rank each of the feature from high correlation to low as $\mathrm{C} 1, \mathrm{C} 2, \mathrm{C} 3, \mathrm{C} 4 \ldots \mathrm{Cn}$.

iii. Information Gain of each of the feature is divided into 3 Sets as group 1 as IG-1, group 2 as IG-2 and similarly group 3 as IG-3 and Correlation is divided into group 1 (CR-1), group 2 (CR-2), group 3 (CR-3), where first set contains features in the range from 1 to 10 and second set has feature from 11 to 30 and rest in 3 set.

iv. Now perform union between IG-1and CR-1 and intersection between (IG-2 and CR-2 to have new set of features, features from IG-3 and CR-3 are removed from dataset.

Step 3: BPNN is then applied to train the reduced feature sets for the detection of anomalies in the network, Since Back Propagation is a confined optimization training method which 
calls a gradient descent method, hence for the optimization of weights applied on the input layer Ant Colony optimization is applied to have continuous optimization process of training in neural networks and reduces error rate.

a) Consider a neural network containing ' 1 ' number of connection weights computed as:

$$
\mathrm{l}=\mathrm{n}_{\mathrm{h}}\left(\mathrm{n}_{\mathrm{i}}+1\right)+\mathrm{n}_{\mathrm{o}}\left(\mathrm{n}_{\mathrm{h}}+1\right)
$$

Where, $n_{h}, n_{i}$ and $n_{o}$ is the number of available hidden, input and output units.

b) Split each of the connection weights $w_{z}$ into $d$ discrete points, where $\in \mathrm{w}_{\mathrm{z}}$ and the range of each $\mathrm{d}$ point are between $\left[\mathrm{w}_{\min }, \mathrm{w}_{\max }\right]$.

c) Initialize Ants and pick one discrete point that belongs to relation weight for each ant ' $a$ ' and store the tag of that discrete point.

d) For all ants $a \in w_{z}$ they began depositing pheromone afterassigning connection weight $\&$ each alliance weight associated with pheromone chart.

e) At what time an ant ' $a$ ' reaches to a correlation weight, the possibility of opt for a discrete point is determined, and the process is repeated until all connection weights have been selected.

$$
p_{i}^{z}=\frac{\tau_{i}^{z}}{\sum_{1=j}^{v} \tau_{j}^{z}}
$$

Where, $v$ stands for the amount of discrete points $a_{i}^{z}$ and $\tau_{i}^{z}$ correspond to the presented pheromone trail of connection weight $w_{i}$ for distinct point $a_{i}^{z}$.

f) Finally when termination points reached and weights are selected, apply activate function and compute error.

g) If (ERROR is large), apply Weight changes optimized by activation function and repeat.

h) The Neural Network can determine whether the Node is NORMAL or MALICIOUS based on the values of the features given. If the node is NORMAL, IDS will continue to monitor it. If the node is MALICIOUS, IDS will utilize the MALICIOUS Node management protocol to remove/block the infected Node from the network, preventing it from causing any harm to network resources or other nodes.

\section{DISCUSSION AND RESULT ANALYSIS}

In this work we are using the concept of information gain and correlation to reduce the data set of KDD-99. Information gain is nothing but the useful information available in the feature called entropy[67,52,56]. Correlation is identified on the basis of dependency among features. As far as KDD-99 dataset is concerned, the KDD99 dataset consists of normal and four classes known as DDoS, R2L, R3, U1 and Probe with diversity and imbalance instances.

$10 \%$ of KDD-99 comprises 494,020 instances with 97,277 normals, while 4,107 probes, 1127 belong to the R2L and only 52 to U2R; remaining 3, 91, 457 being DoS instances. In table 1 , features of KDD-99 are ranked based on information gain and arranged in three group based on amount of information available. IG-1 contains more information compared to IG-2 and IG-3.

\begin{tabular}{|c|c|}
\hline $\begin{array}{l}\text { Group based on } \\
\text { Information Gain }\end{array}$ & Features List \\
\hline $\begin{array}{l}\text { IG1 } \\
\text { [rank =1 to } 10]\end{array}$ & $\begin{array}{l}\text { 'is_guest_login', 'dst_host_srv_rerror_rate', } \\
\text { dst_host_rerror_rate', } \\
\text { 'dst_host_srv_serror_rate', } \\
\text { 'dst_host_serror_rate', } \\
\text { 'dst_host_srv_diff_host_rate', 'su_attempted', } \\
\text { 'num_root', 'urgent' }\end{array}$ \\
\hline $\begin{array}{l}\text { IG2 } \\
\text { [rank =11 to 30] }\end{array}$ & $\begin{array}{l}\text { 'service', 'dst_host_diff_srv_rate', } \\
\text { 'num_failed_logins', 'logged_in', 'dst_bytes', } \\
\text { 'hot', 'duration', 'protocol_type', 'flag', } \\
\text { 'src_bytes', 'land', 'wrong_fragment', } \\
\text { 'dst_host_same_src_port_rate', } \\
\text { 'srv_serror_rate', 'dst_host_same_srv_rate', } \\
\text { 'diff_srv_rate', 'count', 'num_shells', } \\
\text { 'srv_rerror_rate' }\end{array}$ \\
\hline $\begin{array}{l}\text { IG3 } \\
\text { [rank =31 to 42] }\end{array}$ & $\begin{array}{l}\text { 'srv_diff_host_rate', 'dst_host_srv_count', } \\
\text { 'num_access_files', 'num_outbound_cmds', } \\
\text { 'serror_rate', 'num_file_creations', } \\
\text { 'num_compromised', 'same_srv_rate', } \\
\text { 'dst_host_count', 'srv_count', 'is_host_login' }\end{array}$ \\
\hline
\end{tabular}

\section{Table 1. Ranking Based on Information Gain}

The correlation ranking done as CR-1, CR-2 and CR-3 and features come under group is shown in table 2 .

\begin{tabular}{|c|c|}
\hline $\begin{array}{l}\text { Group based on } \\
\text { Correlation }\end{array}$ & Features List \\
\hline $\begin{array}{c}\text { CR-1 } \\
{[\text { rank }=1 \text { to } 10]}\end{array}$ & $\begin{array}{l}\text { 'srv_serror_rate', } \\
\text { 'dst_host_serror_rate', } \\
\text { 'rerror_rate', } \\
\text { 'dst_host_rerror_rate', } \\
\text { 'srv_count' }\end{array}$ \\
\hline $\begin{array}{c}\text { CR-2 } \\
{[\text { rank }=11 \text { to } 30]}\end{array}$ & $\begin{array}{l}\text { 'same_srv_rate', 'dst_host_same_srv_rate', 'hot', 'dst_host_same_src_port_rate', } \\
\text { 'logged_in', } \\
\text { 'diff_srv_rate', 'dst_host_count', 'root_shell', } \\
\text { 'duration', 'srv_diff_host_rate', 'su_attempted', } \\
\text { 'dst_host_diff_srv_rate', 'num_root', } \\
\text { 'dst_host_srv_diff_host_rate', 'urgent', } \\
\text { 'dst_host_srv_serror_rate', 'num_file_creations', } \\
\text { 'num_failed_logins', 'is_guest_login' }\end{array}$ \\
\hline $\begin{array}{c}\text { CR-3 } \\
\text { [rank }=31 \text { to } 42\end{array}$ & $\begin{array}{l}\text { 'land', 'wrong_fragment', 'num_shells', } \\
\text { 'is_host_login', 'dst_bytes', 'src_bytes' }\end{array}$ \\
\hline
\end{tabular}

\section{Table 2. Ranking Based on Correlation}

The combination of IG- $1 \cup$ CR- 1 and common features of IG$2 \cap \mathrm{CR}-2$ are the result of reduced dataset of KDD-99 that contains 24 features. The reduced features are: dst_host_srv_count, srv_rerror_rate, rerror_rate, dst_host_same_src_port_rate, dst_host_diff_srv_rate, is_guest_login, duration, dst_host_srv_diff_host_rate, num_compromised, num_root, dst_host_same_srv_rate, srv_serror_rate, num_failed_logins, dst_host_serror_rate, dst_host_srv_rerror_rate, srv_count, diff_srv_rate, hot, urgent, su_attempted, dst_host_rerror_rate, serror_rate, logged_in, 
dst_host_srv_serror_rate.

Then back propagation neural network designed to train the network and predict the attack types. The system's True positive rate, false positive rate, accuracy, and recall have also been used to evaluate results. To evaluate the classifier's accuracy, confusion matrix or error matrix is used the columns represent the classes and the rows of the confusion matrix. Values of True $(\mathrm{T})$, False $(\mathrm{F})$, and Negative $(\mathrm{N})$ are clearly defined. Tuned PR Fusion (TN is a correct prediction of TN attack) $\mathrm{TN}$ is a correct prediction of classifier if the test instances are normal. Attackers get classified as false positives, but are not detected as such. It is a false negative classifier since it is a normal but attacks are made on it.

\subsection{Results on KDD-99 Dataset}

Table 3 shows the comparison among existing methods, the number of features used in KNN, Naïve Bayes and Multi Level Perceptron [26] is 6, while ANN classifier used 25 features [30], PSO-BP [33] uses all 41 features and proposed method worked on 24 features.

\section{Table 3. Comparison with methods (KDD-99)}

\begin{tabular}{|l|c|c|c|c|c|c|}
\hline Method & Features & TPR & FPR & PR & ACC & F1 \\
\hline $\begin{array}{l}\text { KNN } \\
{[26]}\end{array}$ & 6 & 98.9 & 1.2 & 98.9 & 99 & 98.9 \\
\hline $\begin{array}{l}\text { Naïve } \\
\text { Bayes } \\
{[26]}\end{array}$ & 6 & 93.3 & 6.7 & 93.3 & 93 & 98.3 \\
\hline $\begin{array}{l}\text { MLP } \\
{[26]}\end{array}$ & 6 & 96.5 & 3.5 & 96.5 & 97 & 96.5 \\
\hline $\begin{array}{l}\text { ANN } \\
\text { Classifier } \\
{[30]}\end{array}$ & 25 & 98.8 & 1.2 & 98 & 97 & 98.4 \\
\hline $\begin{array}{l}\text { PSO-BP } \\
{[33]}\end{array}$ & 41 & 98.24 & 1.76 & 98.2 & 98 & 98.22 \\
\hline Proposed & 24 & 98.99 & 1.1 & 99.1 & 99.1 & 99.05 \\
\hline
\end{tabular}

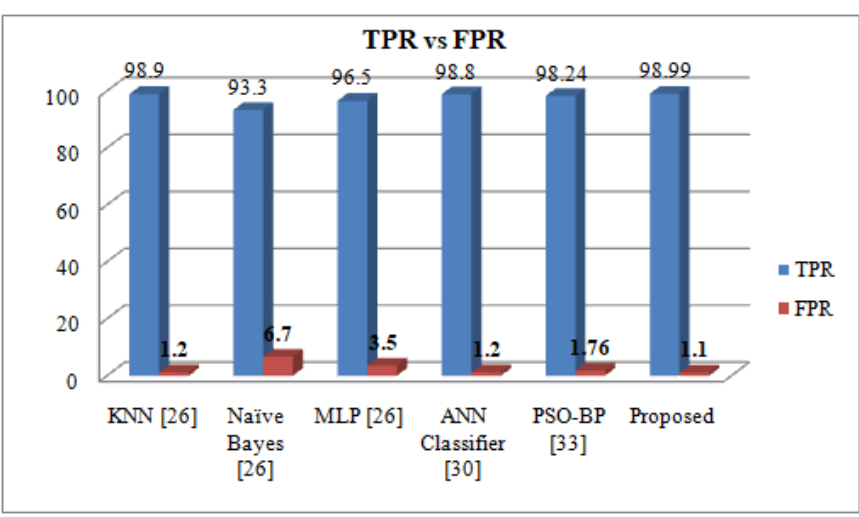

Figure 3: Performance comparison between TPR and FPR (KDD-99)

Figure 3 shows the performance analysis between correctly classified (True Positive Rate) and incorrectly classified (False Positive Rate). In the above figure, KNN and the proposed model is showing the higher TPR and lower FPR.

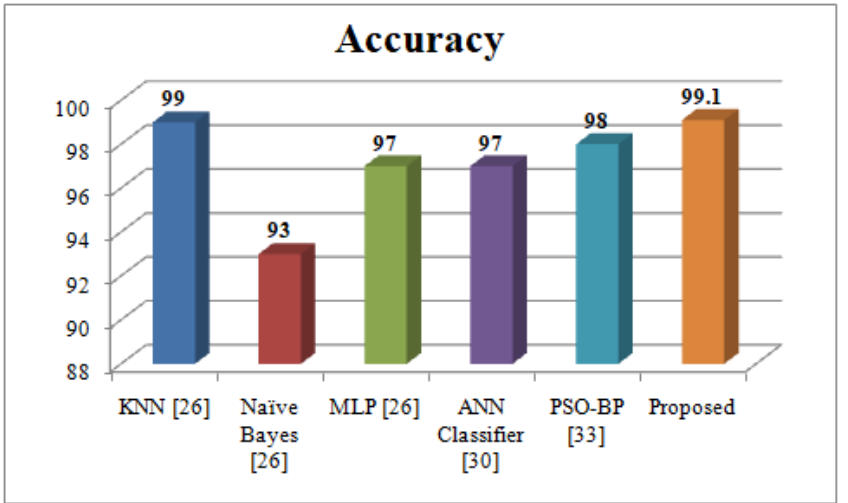

Figure 4: Accuracy of methods (KDD-99)

Figure 4 shows the accuracy obtained by the various methods mentioned in the figure. The highest accuracy rate gained by the KNN and proposed method.

\subsection{Results on NSL-KDD Dataset}

Table 4 shows the comparison among existing methods with NSL-KDD dataset.

\section{Table 4. Comparison with methods (NSL-KDD)}

\begin{tabular}{|l|c|c|c|c|c|c|}
\hline Method & Features & TPR & FPR & PR & ACC & F1 \\
\hline $\begin{array}{l}\text { KNN } \\
{[26]}\end{array}$ & 6 & 99.7 & 0.3 & 96.5 & 98.5 & 98.07 \\
\hline $\begin{array}{l}\text { Naïve } \\
\text { Bayes } \\
{[26]}\end{array}$ & 6 & 95.2 & 4.8 & 93.7 & 94.2 & 94.4 \\
\hline MLP [26] & 6 & 97.5 & 2.5 & 95.6 & 97.8 & 96.54 \\
\hline $\begin{array}{l}\text { ANN } \\
\text { Classifier } \\
{[30]}\end{array}$ & 25 & 99.1 & 0.9 & 96.1 & 98.3 & 97.6 \\
\hline $\begin{array}{l}\text { PSO-BP } \\
\text { [33] }\end{array}$ & 41 & 98.5 & 1.5 & 97.7 & 97.85 & 98.1 \\
\hline Proposed & 24 & 99.8 & 0.2 & 98.9 & 98.99 & 99.35 \\
\hline
\end{tabular}

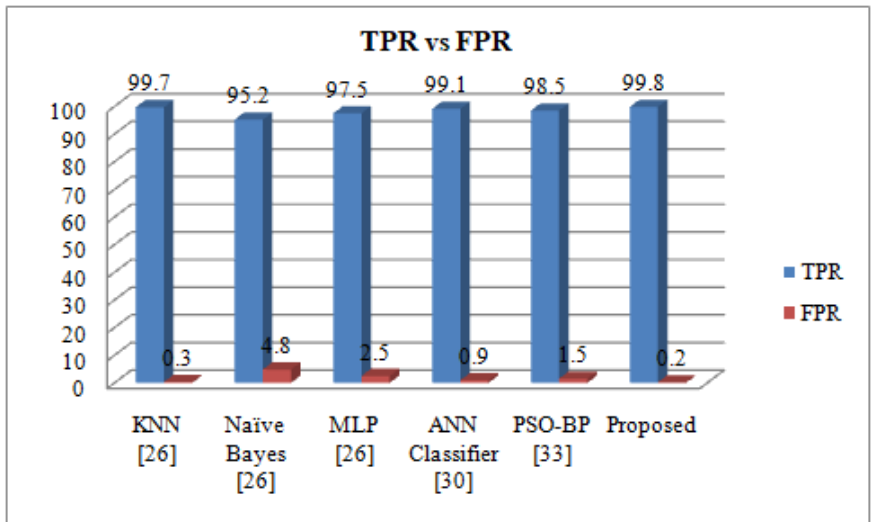

Figure 5: Performance comparison between TPR and FPR (NSLKDD)

Figure 5 shows the performance analysis between TPR and FPR. The KNN and the proposed model is showing the higher TPR and lower FPR. 


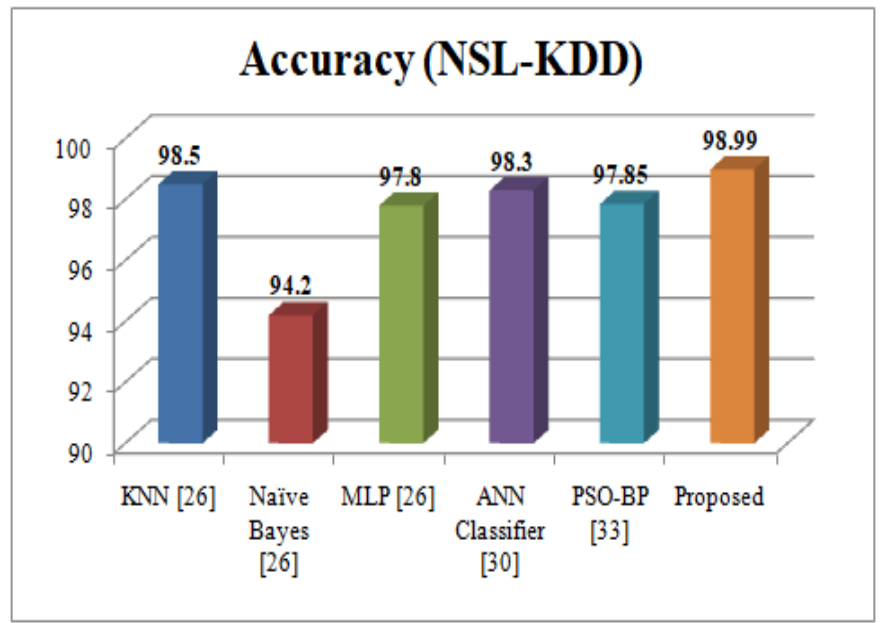

Figure 6: Accuracy of methods (NSL-KDD)

Figure 6 shows the accuracy obtained by the various methods mentioned in the figure using NSL-KDD dataset. The highest accuracy rate gained by the KNN, ANN classifier and proposed method.

\section{CONCLUSION}

Selection of relevant features and processing of unlabeled data are the two major challenges that must be solved for developing an effective IDS model. The unlabeled nature of intrusion detection datasets makes the process of classifying a sample as malicious or normal typical. Dataset Preprocessing, Classification and Performance Evaluation are important phases of their implementation. This research aims to design a new intelligent intrusion detection method based on a more optimized set of features.

The proposed system selects the relevant features based on information gain and correlation among features. The feature reduced dataset is provided to EBPA for training and testing. Results show that the Accuracy, F1 Score and TPR in the proposed technique are improved; the FPR diminishes. This reflects the effectiveness of the proposed technique, compared to other models.

\section{REFERENCES}

[1] J. Jabez and B. Muthukumar "Intrusion Detection System (IDS): Anomaly Detection using Outlier Detection Approach", Proceedings of International Conference on Intelligent Computing, Communication and Convergence (ICCC - 2015), published under Procedia Computer Science, Volume 48, Page 338 - 346, Science Direct, ELSEVIER, 2015.

[2] V. Mane and S. Pawar, "Anomaly based IDS using Back Propagation Neural Network", International Journal of Computer Applications (IJCA) [0975 - 8887], Volume 136, Number 10, Page 29 - 34, February 2016.

[3] Rais, Helmi Md, and Tahir Mehmood, "Dynamic Ant Colony System with Three Level Update Feature Selection for Intrusion Detection". International Journal of Network Security, 20(1), pp. 184-192, 2018.

[4] R. Sharma, H. Kalita and P. Borah, "Analysis of Machine Learning Techniques based Intrusion Detection Systems", Proceedings of 3rd International Conference on Advanced Computing, Networking and Informatics, published under Smart Innovation, Systems and
Technologies, Volume 44, Page 485 - 493, SPRINGER 2016.

[5] Javadzadeh, G., \& Azmi, R.: IDuFG: Introducing an Intrusion Detection using Hybrid Fuzzy Genetic Approach. IJ Network Security, 17(6), 754770. (2015).

[6] Sonawane, S., Pardeshi, S., \& Prasad, G.: A survey on intrusion detection techniques. World journal of science and technology, 2(3), 127-133. (2012).

[7] M. Aljanabi, M. Ismail and A. Ali, "Intrusion Detection Systems, Issues, Challenges and Needs", International Journal of Computational Intelligence Systems, Volume 14, Issue 1, Page 560 - 571, Atlantis Press, 2021.

[8] H. Kour and N. Gondhi, "Machine Learning Techniques: A Survey", Proceedings of ICIDCA - 2019, published under LNDECT, Volume 46, Page 266 - 275, SPRINGER, 2020.

[9] KDD Cup $1999 . \quad$ Available on: http://kdd.ics. uci.edu/databases/kddcup99/kddcup99.html, accessed on jan 2021.

[10] NSL-KDD dataset. Available on: http://nsl.cs.unb.ca/NSL-KDD/, accessed on Sept. 2021.

[11] J. Cai, J. Luo, S. Wang and S. Yeng, "Feature Selection in Machine Learning: A New Perspective", Journal of Neuro-Computing, Volume 300, Page 70 - 79, ELSEVIER, 2018.

[12] Goen, Anjana \& Tiwari, D.C., "Pattern Recognition of Individual and Combined Fingers Movements Based Prosthesis Control Using Surface EMG Signals", International Journal of Electrical \& Electronics Research. (IJEER) Volume 3, Issue 4, Pages 70-78, December 2015.

[13] Dubey, G. P., \& Bhujade, R. K.: Optimal feature selection for machine learning based intrusion detection system by exploiting attribute dependence. Materials Today: Proceedings, (2021). https://doi.org/10.1016/j. matpr.2021.04.643

[14] P. Shen, X. Ding, W. Ren and S. Liu, "A Stable Feature Selection Method based on Relevancy and Redundancy", Proceedings of SCSET 2020, published under Journal of Physics: Conference Series, Volume 1732, IOP Publishing, 2021.

[15] H. Lim and D. Kim, "Pair-wise Dependence-based Unsupervised Feature Selection", Journal of Pattern Recognition, Volume 111, ELSEVIER, 2021.

[16] N. Mishra and P. Singh, "FS - MLC: Feature Selection for Multi Label Classification using Clustering in Feature Space", Journal of Information Processing and Management, Volume 57, ELSEVIER, 2020.

[17] Kumar, Koushal, and Jaspreet Singh Batth. "Network intrusion detection with feature selection techniques using machine-learning algorithms." International Journal of Computer Applications, 150, no. $12,2016$.

[18] Dua, Mohit. "Attribute selection and ensemble classifier based novel approach to intrusion detection system." Procedia Computer Science, 167, pp. 2191-2199, 2020.

[19] N. Neupane and S. Shakya, "Comparative Analysis of Back Propagation Algorithm Variants for Network Intrusion Detection", Proceedings of International Conference on Computing, Communication and Automation (ICCCA), Page 726 - 729, IEEE, 2017.

[20] X. Lu, D. Han, L. Duan and Q. Tian, "Intrusion Detection of Wireless Sensor Networks based on IPSO Algorithm and BP Neural Network", International Journal of Computational Science and Engineering, Volume 22, Page 221 - 232, INDERSCIENCE, 2020.

[21] L. Tao, Y. Jun and S. Qian, "Intrusion Detection Algorithm of EPSO combined with BP Neural Network", Proceedings of International Conference on Intelligent Transportation, Big Data and Smart City (ICITBS), Page 893 - 896, IEEE, 2020.

[22] H. Mustafidah and Suwarsito, "Performance of Levenberg Marquardt Algorithm in Back Propagation Network based on the number of Neurons in Hidden Layer and Learning Rate", JUITA: Journal Informatica, Volume 8, Number 1, Page 29-35, May 2020.

[23] Karatas, Gozde, and Ozgur Koray Sahingoz. "Neural network based 
intrusion detection systems with different training functions." In 2018 6th International Symposium on Digital Forensic and Security (ISDFS), pp. 1-6. IEEE, 2018.

[24] Alhaj, Taqwa Ahmed, Maheyzah Md Siraj, Anazida Zainal, Huwaida Tagelsir Elshoush, and Fatin Elhaj. "Feature selection using information gain for improved structural-based alert correlation." PloS one 11, no. 11,: $\quad$ e0166017, $2016 . \quad$ Online available: https://journals.plos.org/plosone/article?id=10.1371/journal.pone.016601

[25] Shahbaz, Mahsa Bataghva, Xianbin Wang, Aydin Behnad, and Jagath Samarabandu. "On efficiency enhancement of the correlation-based feature selection for intrusion detection systems." In 2016 IEEE 7th Annual Information Technology, Electronics and Mobile Communication Conference (IEMCON), pp. 1-7, 2016

[26] Salih, Azar Abid, and Maiwan Bahjat Abdulrazaq. "Combining best features selection using three classifiers in intrusion detection system." In 2019 IEEE International Conference on Advanced Science and Engineering (ICOASE), pp. 94-99, 2019.

[27] Zhang, Yong, Xuezhen Ren, and Jie Zhang. "Intrusion detection method based on information gain and ReliefF feature selection." In 2019 IEEE International Joint Conference on Neural Networks (IJCNN), pp. 1-5, 2019.

[28] Chormunge, Smita, and Sudarson Jena. "Efficient Feature Subset Selection Algorithm for High Dimensional Data." International Journal of Electrical \& Computer Engineering, 6, no. 4, pp.: 2088-8708, 2016.

[29] Bansal, Ashu, and Sanmeet Kaur. "Data dimensionality reduction (DDR) scheme for intrusion detection system using ensemble and standalone classifiers." In International Conference on Advances in Computing and Data Sciences, pp. 436-451. Springer, Singapore, 2019.

[30] Manzoor, Ishfaq, and Neeraj Kumar. "A feature reduced intrusion detection system using ANN classifier." Expert Systems with Applications, 88, pp: 249-257, 2017.

[31] Singh, Harendra, and Roop Singh Solanki. "Classification \& Feature extraction of Brain tumor from MRI Images using Modified ANN Approach." International Journal of Electrical \& Electronics Research. (IJEER) Volume 9, Issue 2, Pages 10-15, May 2021.

[32] R. Liu, "A Computer Network Intrusion Detection Technology based on Improved Neural Network Algorithm", Journal of Telecommunications and Radio Engineering, Volume 79, Issue 7, Page 593 - 601, 2020.

[33] Goel, A., Bhujade, R.K.,"A functional review, analysis and comparison of position permutation based image encryption techniques" (2020) International Journal of Emerging Technology and Advanced Engineering, 10 (7), pp. 97-99.

[34] Adytia, N.R., Kusuma, G.P. "Indonesian license plate detection and identification using deep learning", (2021) International Journal of Emerging Technology and Advanced Engineering, 11 (7), pp. 1-7.

[35] Rahman, R.A., Masrom, S., Zakaria, N.B., Halid, S. "Auditor choice prediction model using corporate governance and ownership attributes: Machine learning approach", (2021) International Journal of Emerging Technology and Advanced Engineering, 11 (7), pp. 87-94.

[36] Rahman, A.S.A., Masrom, S., Rahman, R.A., Ibrahim, R.,"Rapid software framework for the implementation of machine learning classification models", (2021) International Journal of Emerging Technology and Advanced Engineering, 11 (8), pp. 8-18.

[37] Oleksenko, O., Khudov, H., Petrenko, K., Horobets, Y., Kolianda, V., Kuchuk, N., Konstantinov, A., Kireienko, V., Serdiuk, O., Yuzova, I., Solomonenko, Y. "The development of the method of radar observation system construction of the airspace on the basis of genetic algorithm", (2021) International Journal of Emerging Technology and Advanced Engineering, 11
(8), pp. 23-30.

[38] Al-Thani, M.G., Yang, D.,"Machine Learning for the Prediction of Returned Checks Closing Status", (2021) International Journal of Emerging Technology and Advanced Engineering, 11 (6), pp. 19-26.

[39] Kurnia, R.I., Girsang, A.S., "Classification of user comment using word2vec and deep learning", (2021) International Journal of Emerging Technology and Advanced Engineering, 11 (5), pp. $1-8$.

[40] Meshram, S., Kumar, S., Shukla, S.,"Enhanced robust and invisible of digital image using discrete cosine transform technique and binary shifting technique", (2020) International Journal of Emerging Technology and Advanced Engineering, 10 (10), pp. 113-118.

[41] Choudhary, D., Malasri, S. "Machine learning techniques for estimating amount of coolant required in shipping of temperature sensitive products", (2020) International Journal of Emerging Technology and Advanced Engineering, 10 (10), pp. 67-70.

[42] Chakraborty, R., Sanyal, S., Das, P., "IoT based thermal signature detector with alarm \& e-mail notification with integrated social gathering screening using computer vision", (2020) International Journal of Emerging Technology and Advanced Engineering, 10 (4), pp. 164-171.

[43] Nahar, A., Sharma, S., "Machine learning techniques for diabetes prediction: A review", (2020) International Journal of Emerging Technology and Advanced Engineering, 10 (3), pp. 28-34.

[44] Vijayalakshmi, K., "Comparitive approach of data mining for diabetes prediction and classification", (2020) International Journal of Emerging Technology and Advanced Engineering, 10 (2), pp. 19-26.

[45] Gani, A.S.F., Miskon, M.F., Hamzah, R.A., Mohamood, N., Manap, Z., Zulkifli, M.F., Shah, M.A.M.A.S. , "A live-video automatic number plate recognition (anpr) system using convolutional neural network (CNN) with data labelling on an android smartphone", (2021) International Journal of Emerging Technology and Advanced Engineering, 11 (10), pp. 88-95.

[46] Michael, C., Utama, D.N.,"Social media based decision support model to solve Indonesian waste management problem: An improved version", (2021) International Journal of Emerging Technology and Advanced Engineering, 11 (10), pp. 1-12.

[47] Khudov, H., Oleksenko, O., Lukianchuk, V., Herasymenko, V., Yaroshenko, Y., Ishchenko, O., Ikaiev, D., Golovchenko, O., Volobuiev, A., Drob, Y., Solomonenko, Y., Khizhnyak, I. , "The determining the flight routes of unmanned aerial vehicles groups based on improved ant colony algorithms", (2021) International Journal of Emerging Technology and Advanced Engineering, 11 (9), pp. 23-32.

[48] Rahman, R.A., Masrom, S., Zakaria, N.B., Nurdin, E., Abd Rahman, A.S.,"Prediction of earnings manipulation on Malaysian listed firms: A comparison between linear and treebased machine learning", (2021) International Journal of Emerging Technology and Advanced Engineering, 11 (8), pp. 111-120.

[49] Vorapracha, P. "Potable water order forecasting system using data mining technique" (2021) International Journal of Emerging Technology and Advanced Engineering,11 (6),pp.1-8.

[50] Muqodas, A.U., Kusuma, G.P.,"Promotion scenario based sales prediction on E-retail groceries using data mining", (2021) 
International Journal of Emerging Technology and Advanced Engineering, 11 (6), pp. 9-18.

[51] Khan, R., Pillai, B.,"Enhanced digital image data hiding using image encrypted block histogram shifting method and ardiem", (2020) International Journal of Emerging Technology and Advanced Engineering, 10 (7), pp. 41-46.

[52] Obowu, C.D., Abam, T.K., Ngah, S.,"Semi - Automatic detection and monitoring of surface water anomaly in offshore oil mining lease 59 in Nigeria", (2020) International Journal of Emerging Technology and Advanced Engineering, 10 (1), pp. 32-48.

[53] Vu Ngoc Son, "Optimizing Network Anomaly Detection Based on Network Traffic", (2021) International Journal of Emerging Technology and Advanced Engineering, 11 (11), pp. 53-60.

[54] Vu Ngoc Son, "Developing a Framework for Detecting Phishing URLs using Machine Learning”, (2021) International Journal of Emerging Technology and Advanced Engineering, 11 (11), pp. 61-67.

[55] Enrique Lee Huamaní, Lilian Ocares-Cunyarachi,"Use of Artificial Intelligence for Face Detection with Face Mask in Real Time to Control the Entrance to an Entity", (2021) International Journal of Emerging Technology and Advanced Engineering, 11 (11), pp. 68-75.

[56] Yesica Pamela Leandro Chacon, Omar ChamorroAtalaya,"Automatic Fire Detection and Extinction with Infrared Multispectrum Electro-Optical Technology with Watch-Dog Timer Control, for a $220 \mathrm{KV}$ to $33 \mathrm{KV}$ Transformer", (2021) International Journal of Emerging Technology and Advanced Engineering, 11 (11), pp101-113

[57] Brian Meneses-Claudio, Luis Nuñez-Tapia, Witman AlvaradoDíaz, Alicia Alva Mantari,"Implementation of a Thermal Image Processing System to Detect Possible Cases of Patients with COVID-19", (2021) International Journal of Emerging Technology and Advanced Engineering, 11 (11), pp 130-139

[58] Sebastian Ramos-Cosi, Natalia I. Vargas-Cuentas, " Prototype of a System for Quail Farming with Arduino Nano Platform, DHT11 and LM35 Sensors, in Arequipa, Peru", (2021) International Journal of Emerging Technology and Advanced Engineering, 11 (11), pp 140-146.

[59] Mohammad Taleghani, Ataollah Taleghani, "The Analysis and Evaluation of profitability in Accepted Companies in Tehran Stock Exchange with ROA and ROE Approaches in Industrial Companies", (2021)International Journal of Recent Development in Engineering and Technology(IJRDET), ISSN 2347-6435, 10 (2), pp 38-46.

[60] Dr. Meghna Utmal, "Taxonomy on Machine Learning Algorithms",(2021) International Journal of Recent Development in Engineering and Technology(IJRDET), ISSN 2347-6435, 10 (8), pp 1-7.

[61] Sai Prabhath Sarma, MD Abu Sufiyan, Prathyush Kumar, "Operator Authentication Using Facial Recognition", (2021) International Journal of Recent Development in Engineering and Technology, ISSN 2347-6435, 10 (7), pp7-10.

[62] Mohammad Hossein Doost Mohammadi, Arman Garousi, “A Fast and Simple Face Detection Algorithm Using Neural Network and Its Implementation on FPGA",(2021) International Journal of Recent Development in Engineering and Technology , ISSN 2347-6435, 10 (5), pp 10-20.

[63] Md. Qaiser Abdulhaque, Saheb Halder, R K Sharma, Saroj Mahapatra, Dr. Shambhu Nath Mishra, Dr. Amit Dhorde,
"Impact Assessment of Narwa Programme Through Remote Sensing (RS) and Geographical Information System (GIS)"'(2021) International Journal of Recent Development in Engineering and Technology, ISSN 2347-6435, 9(12), pp 1-4.

[64] Dubey, G.P., Bhujade, R.K. "Improving the performance of intrusion detection system using machine learning based approaches", International Journal of Emerging Trends in Engineering Research,2020, 8(9), pp. 4947-4951

[65] Goel, A., Bhujade, R., "A prototype model for image encryption using zigzig blocks in inter-pixel displacement of RGB value" International Journal of Advanced Trends in Computer Science and Engineering, 2020, 9(5), pp. 6905-6912

[66] Goel, A., Bhujade, R., "A functional review of image encryption techniques" ,International Journal of Scientific and Technology Research, 2019, 8(9), pp. 1203-1205

[67] Sharma, Suchi, and Anjana Goen. "Comparison and Performance Evaluation of Low Pass Filter Design for various samples using Artificial Neural Network." International Journal of Electrical \& Electronics Research. (IJEER) Volume 4, Issue

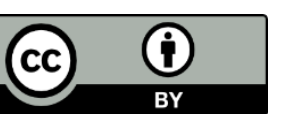
3, Pages 74-79, September 2016.

(C) 2021 by the Prof. Ghanshyam Prasad Dubey, Dr. Rakesh Kumar Bhujade. Submitted for possible open access publication under the terms and conditions of the Creative Commons Attribution (CC BY) license (http://creativecommons.org/licenses/by/4.0/). 